\title{
Validation of screening questions and symptom coherence of night eating in the Swedish Twin Registry
}

\author{
Kelly C. Allison ${ }^{\mathrm{a}, *}$, Jennifer D. Lundgren ${ }^{\mathrm{b}}$, Albert J. Stunkard ${ }^{\mathrm{a}}$, Cynthia M. Bulik ${ }^{\mathrm{c}, \mathrm{d}}$, \\ Anna Karin Lindroos ${ }^{\mathrm{e}}$, Laura M. Thornton ${ }^{\mathrm{c}}$, Finn Rasmussen ${ }^{\mathrm{f}, \mathrm{g}, *}$ \\ ${ }^{\text {a }}$ Perelman School of Medicine, University of Pennsylvania Center for Weight and Eating Disorders, Philadelphia, PA 19104-3309, USA \\ ${ }^{\mathrm{b}}$ Department of Psychology, University of Missouri-Kansas City, Kansas City, MO 64116, USA \\ ${ }^{\mathrm{c}}$ Department of Psychiatry, University of North Carolina at Chapel Hill, Chapel Hill, NC 27599, USA \\ ${ }^{\mathrm{d}}$ Department of Nutrition, University of North Carolina at Chapel Hill, Chapel Hill, NC 27599, USA \\ ${ }^{\mathrm{e}}$ Risk Benefit Assessment Department, National Food Agency, SE-751 26 Uppsala, Sweden \\ ${ }^{\mathrm{f}}$ Department of Public Health Sciences, Karolinska Institutet Widerström Building, SE-17177 Stockholm, Sweden \\ ${ }^{\mathrm{g}}$ Centre for Epidemiology and Community Medicine Stockholm County Council, Health Care Services, SE-17177 Stockholm, Sweden
}

\begin{abstract}
Background: Screening criteria have been used to estimate the prevalence of night eating syndrome (NES), but no validation studies have been conducted.

Method: We examined the validity of two screening questions for NES using a structured interview with adults enrolled in the Swedish Twin Study of Adults: Genes and Environment (STAGE) study. We also examined the coherence of the proposed diagnostic criteria for NES. A total of 416 participants $(m e n=179$; women $=237)$ completed the interview and reported complete data for study inclusion.

Results: The following values were calculated for the screening items after confirmation by interview for men and women, respectively: positive predictive value $=.66$ and .67 , negative predictive value $=.52$ and .45 , sensitivity $=.62$ and .63 , and specificity $=.56$ and .50 . As increasingly stringent diagnostic criteria were applied to the sample, prevalence of NES dropped. Nocturnal ingestions were more likely to co-occur with other NES symptoms than evening hyperphagia, which occurred frequently, but often in isolation; women were more likely to report a co-occurrence of symptoms than men, who were not likely to report distress related to NES symptoms.

Conclusion: In sum, almost two-thirds of participants were correctly identified as having NES (without considering distress or impairment) if they answered positively on the screening questions, and about half of the participants were correctly identified as not having NES when answering negatively on the screening questions. Although self-report questions are somewhat informative, a structured interview remains the gold standard for diagnosing NES.
\end{abstract}

(C) 2014 Published by Elsevier Inc.

\section{Introduction}

Although night eating syndrome (NES) was first described over fifty years ago [1], research to promote valid and reliable assessment tools have lagged. The challenge for many years was the lack of a uniform set of diagnostic criteria for NES, which has made direct

* Corresponding authors. A.J. Stunkard is to be contacted at: 3535 Market St., 3rd Floor, Philadelphia, PA 19104-3309. Tel.: +1 215898 7314; fax: +1 215898 2878. F. Rasmussen, Department of Public Health Sciences, Karolinska Institutet, Norrbacka, SE-17176 Stockholm, Sweden. Tel.: +46 852480181; fax: +46 8308008.

E-mail addresses: kca@mail.med.upenn.edu (K.C. Allison), finn.rasmussen@ki.se (F. Rasmussen). comparisons of prevalence estimates across studies difficult. NES has only recently been included in the Diagnostic and Statistical Manual of Mental Disorders (DSM 5) [2] within the Feeding and Eating Disorders Not Elsewhere Classified category. It is not specifically named in the World Health Organization's International Classification of Diseases (ICD-10) [3]. An expert panel convened in 2008 to propose a set of diagnostic criteria for NES to advance research in this field [4] to help standardize the definition of NES.

NES is defined by three core criteria. Criterion A is characterized by a delay in the circadian pattern of eating such that, 1) at least one quarter of daily caloric intake is consumed after the evening meal (evening hyperphagia), and/or 2) waking to eat (nocturnal ingestions) occurs at least twice per 
week [4]. Criterion B states that awareness and recall of the eating episodes must be present to differentiate the behavior from sleep-related eating disorder (SRED), a parasomnia [5]. Criterion $\mathrm{C}$ specifies that at least three of five modifiers must also be present four or more days per week, including 1) morning anorexia or breakfast skipping, 2) a strong urge to eat in the evening or nighttime, 3) sleep onset or sleep maintenance insomnia, 4) belief that one must eat to sleep, and 5) depressed mood that often worsens in the evening.

The only validated assessment instrument for the screening of NES is the Night Eating Questionnaire (NEQ) [6]. This is a 14-item survey, with response options on a Likert scale. Internal consistency in the original validation study was .70 [7], similar to the .79 found in the Spanish translation and .78 found in the Portuguese translation [8]. It has rarely been used in population-based prevalence studies. At least six previous groups have utilized various screening approaches to estimate the prevalence of night eating in general population or large community samples. The first general population estimate was presented by Rand et al. [9]. They interviewed 2097 adults (mean age $=52.8$ years; mean $\mathrm{BMI}=24.9 \mathrm{~kg} / \mathrm{m}^{2}, 58 \%$ female, and $78.5 \%$ white) for the presence of night eating, defined by experiencing morning anorexia, delay of eating after awakening for several hours, excessive evening eating, evening tension and/or feeling upset, and insomnia over the past two months. Criteria for night eating were met by $1.5 \%$ of this sample. Next, StriegelMoore et al. [10] surveyed a cohort of 682 black and 659 white, young women. Their criteria included awakening during the night at least once per week, snacking at least sometimes during these awakenings, consuming at least one half of daily energy intake after dinner, and no eating in the morning until after $9 \mathrm{am}$. The prevalence of night eating using this definition was $1.6 \%$ in this population.

Lamerz et al. [11] surveyed 1979 children (mean age $=$ 5.8 years, $92 \%$ were below 90th percentile for BMI), 1959 mothers (mean age $=35.9$ years, $67 \%$ normal weight, $20 \%$ overweight, and $9 \%$ obese) and 1877 fathers (mean age $=$ 39 years, $51 \%$ normal weight, $39 \%$ obese, and $9 \%$ obese) in an urban, German community population. They asked, "Did you ever notice that you/your child got up late in the evening or during the night to eat large amounts of high calorie food more than once a week for a time period of at least three months?" The prevalence for this definition of night eating for each group was: $1.1 \%$ of children, $5.8 \%$ of mothers, and $4.5 \%$ of fathers. More recently, Striegel-Moore and colleagues [12] examined 24-h food intake data from the Continuing Survey of Food Intakes by Individuals (CSFII; $\mathrm{n}=10,741)$ and the National Health and Nutrition Examination Survey-III (NHANES-III; $\mathrm{n}=18,407$ ) to report a population prevalence of night eating. For both samples, participants were $48 \%$ male and $78 \%$ White. Age groups for the studies were $9 \%$ adolescent; $22 \%$ (CSFII) and $24 \%$ (NHANES-III) young adult, 53\% (CSFII) and 52\% (NHANES-III) adult, and 15\% elderly. They defined night eating behavior three ways, yielding the following preva- lence estimates: a) $31 \%$ of CSFII and $36 \%$ of NHANES-III reported consuming $25 \%$ or more of daily energy intake after 7:00 pm; b) $11 \%$ of CSFII and 13\% of NHANES-III reported consuming $50 \%$ or more of daily energy intake after $7: 00 \mathrm{pm}$; and c) $9 \%$ of CSFII and $12 \%$ of NHANES-III reported any eating after 11:00 pm.

Finally, two questions were used to screen for night eating in the large population cohort, Swedish Twin Study of Adults: Genes and Environment (STAGE) [13]. Participants were asked, "How often do you get up at night to eat?" and "What proportion of your daily food intake takes place after the evening meal?" [13]. Those who reported awakening to eat at least weekly and/or consumed $25 \%$ or more of their energy after the evening meal met the broad definition of night eating. The narrow definition consisted of awakenings with food at least once per week and/or consuming at least $50 \%$ of their energy intake after dinner. For men $(\mathrm{n}=9743$, mean age $=$ 34.7 years, $\mathrm{BMI}=24.6 \mathrm{~kg} / \mathrm{m}^{2}$ ), the prevalence was $4.6 \%$ for broad and $2.5 \%$ for narrow night eating. For women $(\mathrm{n}=$ 11,998 , mean age $=34.5$ years, $\mathrm{BMI}=23.1 \mathrm{~kg} / \mathrm{m}^{2}$ ), the prevalence was $3.4 \%$ for broad and $1.7 \%$ for narrow night eating.

These studies suggest a wide range of prevalence estimates for night eating from $1.5 \%$ up to $35 \%$ in adult populations, depending on the assessment technique and definition used. Additionally, the validity of these brief assessments in identifying those meeting full diagnostic criteria for NES remains unknown. As demonstrated by the various criteria employed in the studies described above, more research is also needed to test the coherence of the proposed diagnostic criteria for NES. Without such data, the clinical utility of the criteria remains in question [14]. The purpose of the current study was to validate the two screening questions used in the STAGE study to identify cases of NES against the gold standard structured psychiatric interview. We interviewed respondents to calculate the positive predictive value, negative predictive value, sensitivity, and specificity of the two screening questions compared to a structured interview based on the Night Eating Syndrome History and Inventory (NESHI) [15], described below. We also sought to examine the cohesion of NES symptoms as reported in this large, population-based sample.

\section{Method}

\subsection{Participants and procedure}

Participants were drawn from the Swedish Twin study of Adults: Genes and Environment study (STAGE; http://ki.se/ $\mathrm{ki} / \mathrm{jsp} /$ polopoly.jsp? $\mathrm{d}=9610 \& \mathrm{l}=\mathrm{en})$, a population-based sample of Swedish twins born 1959-1985 (ages 20-47) [16]. Over 43,000 twins were eligible to participate. The response rate was $59.6 \%$, with more than 25,000 twins responding to some portions of the study. Data for approximately 1300 questions across 34 health and demographic topics were collected through the STAGE website (43.1\% responded exclusively using the web-based format) and by phone 
(16.5\% responded using the telephone format exclusively) in 2005-2006. STAGE is described in detail elsewhere $[16,17]$. The Regional Ethics Committee at the Karolinska Institutet and the Biomedical Institutional Review Boards at the University of Pennsylvania and the University of North Carolina approved the study. All participants provided informed consent.

Individuals were eligible for the current study if they entered the eating disorders section of STAGE and answered either of the night eating screening questions regarding nocturnal ingestions and evening hyperphagia, yielding 22,863 participants $(10,091$ men and 12,772 women). Of these, 911 participants (473 men and 438 women) answered positively to at least one of these screening questions. These 911 participants who screened positive and their co-twins were eligible for the NESHI (described below) [15] to provide a mix of participants with positive and negative responses. Twins who refused further contact with the registry and those with incomplete or missing data for their co-twins were excluded from the interview.

Interviews using the NESHI were conducted by phone between May and December 2007 using a private interview/ fieldwork company (Intervjubolaget-IMRI, Stockholm, Sweden). Interviewers were informed about the study and trained by study personnel (AKL). The training consisted of a four-hour session on how to administer the NESHI interviews, and specific emphasis was given to the open dietary recall part of the interview. In all, 734 participants were contacted for the validation interview (388 who screened positive and 346 co-twins who screened negative). Of these, $627(85.4 \%)$ were successfully interviewed, with $416(56.7 \%)$ providing complete information needed for assessment of primary outcomes in the current study and comprising our sample.

\subsection{Measures}

\subsubsection{Screening questions}

The screening questions for night eating were asked at the end of the eating disorders section. Nocturnal ingestions were assessed with the question: "How often do you get up at night to eat?" Response options were: never, once or twice, weekly, and nightly. Evening hyperphagia was assessed with the question: "What proportion of your daily food intake takes place after the evening meal?" Response options were: $0 \%$ (none), $1 \%-24 \%$ (up to a quarter), $25 \%-49 \%$ (about half); $50 \%-75 \%$ (more than half), and $75 \%-100 \%$ (all). If respondents answered that they got up to eat at night either weekly or nightly to the nocturnal ingestions question and/or they indicated eating at least $25 \%$ of their energy intake after supper, suggesting evening hyperphagia, they were classified positively for screened night eating.

\subsubsection{Validation instruments}

The validation instrument included the NESHI which is a semi-structured clinical interview, developed by Drs.
Allison, Stunkard, and colleagues at the University of Pennsylvania, for the assessment and diagnosis of NES [15]. The validated NEQ is embedded in the NESHI. Specific areas of assessment include typical 24-h pattern of food intake, hunger, sleep, and mood patterns over the previous 28 days, distress (i.e., How upsetting is your night eating to you?) or functional impairment (i.e., How much has your night eating affected your life?), and history and course of night eating symptoms. For the present study, the NESHI was modified to include a dietary recall. The instrument was translated into Swedish and back-translated into English for use with this Swedish population. Trained interviewers completed the NESHI interview by phone with participants, taking up to one hour to administer it.

As part of this modified NESHI interview, each participant completed a multiple pass dietary recall with the trained interviewer for all foods and drinks consumed after dinner the previous day. The recall was confined to intake after dinner to reduce participant burden. We estimated the percentage of energy consumed after dinner (i.e., validation of the evening hyperphagia criterion) from these recall data. First, a dietician calculated energy intake based on the Nutrition Database of the Swedish National Food Agency, using the software Dietist. Second, resting metabolic rate (RMR) for each participant was calculated using the Mifflin-St. Joer equation [18]; this equation has been shown to provide the highest percentage of accurate estimates of RMR across weight categories $(82 \%$ accurate for normal weight and 70\% accurate for obese persons) [19]. Third, the intake estimates from the dietary recall were used to calculate the number of Joules each individual consumed between 8:00 pm and 5:00 am the following morning. To calculate the proportion of total daily energy intake consumed between 8:00 pm and 5:00 am, we divided the number of nighttime calories consumed by the participant's RMR. If the resulting proportion of energy intake were $\geq 25 \%$, the participant was classified as positive for validated evening hyperphagia.

Participants were classified as positive for validated nocturnal ingestions if they reported eating upon awakening at night at least twice per week on the NESHI interview. All others were considered negative for validated night eating.

Full NES was also evaluated using the NES interview. Participants included in this classification consumed at least $25 \%$ of their calories between 8:00 pm and 5:00 am and/or reported eating upon awakening at night at least twice a week. They also endorsed NES Criterion B, awareness, and at least three of the NES Criterion C symptom modifiers [4]: morning anorexia, evening or nighttime cravings, insomnia, belief in needing to eat to sleep, and depression/mood worsening in evening.

\subsubsection{Sociodemographics}

Participants were asked to indicate the highest level of education completed. Participants were grouped according to having completed secondary school or less and those with 
greater than secondary school. Marital/cohabiting status was also assessed.

\subsection{Statistical analyses}

All analyses were performed using SAS/STAT software, Version 9.2 of the SAS System for Windows (Cary, NC). Descriptive analyses were conducted to characterize the sample in regard to screened night eating and validated night eating. Prevalence of screened night eating, validated night eating and night eating syndrome was determined.

False negative, true negative, false positive, true positive, positive predictive value (the proportion of subjects with positive test results who are correctly diagnosed), negative predictive value (the proportion of subjects with a negative test result who are correctly diagnosed), sensitivity (the proportion of actual positives which are correctly identified as such, e.g. the percentage of participants who are correctly identified as having NES), and specificity (the proportion of negatives which are correctly identified, e.g. the percentage of healthy people who are correctly identified as not having NES) were computed based on the screened night eating group assignment and validated night eating group assignment.

Finally, the co-occurrence of NES Criterion A (evening hyperphagia and/or nocturnal ingestions) and Criterion $\mathrm{C}$ (associated features) was evaluated by calculating a conditional probability using the formula $\mathrm{P}(\mathrm{X}$ and $\mathrm{Y}) /$ $\mathrm{P}(\mathrm{Y})$. As was reported in a previous study [20], only the behavioral features of NES (those in Criteria A and C) were used, rather than differential diagnoses (e.g., Criterion $B$ is used to differentiate night eating due to NES versus SRED), course of symptoms, or indicators of distress or functional impairment.

\section{Results}

\subsection{Screening outcomes}

Based on the criteria from the two screening items (eating upon awakening at night at least weekly and/or consuming $25 \%$ or more of their daily energy intake after dinner), 93 $(52.0 \%)$ men and $138(58.2 \%)$ women who completed the validation interview had originally been categorized as positive for night eating. Of the men in the screen positive group, 38 (40.9\%) reported nocturnal ingestions but not evening hyperphagia, 52 (55.9\%) reported evening hyperphagia but not nocturnal ingestions, and $2(2.2 \%)$ reported both items. One (1.1\%) also reported nocturnal ingestions but did respond to the evening hyperphagia item. Of the 138 women in the screen positive group, $43(31.2 \%)$ reported nocturnal ingestions but not evening hyperphagia (with an additional $5(3.6 \%)$ who did not respond to the evening hyperphagia item), 83 (60.1\%) reported evening hyperphagia but not nocturnal ingestions, and 7 (5.1\%) reported both items.

Means (SD) for age at interview, current BMI, education, and marital status by sex and screening group are presented in Table 1. For men, no differences in age at interview (t-value $=-0.62, \mathrm{p}<.54$ ), current BMI $(\mathrm{t}$-value $=-0.48, \mathrm{p}<.64)$, married/cohabiting $\quad\left(\chi^{2}=0.19\right.$, $\mathrm{p}<.67)$ and education $\left(\chi^{2}=0.15, \mathrm{p}<.70\right)$ were observed between the screen positive and screen negative night eating groups. Similarly, no differences were observed between the women in the screen positive and screen negative night eating groups for most variables [age: (t-value $=0.31$, $\mathrm{p}<.77$ ), current BMI (t-value $=1.43, \mathrm{p}<.16$ ) and married/ cohabiting $\left.\left(\chi^{2}=0.82, \mathrm{p}<.37\right)\right]$. However, fewer women in the screen positive group had more than a secondary school education (college) $\left(\chi^{2}=6.45, p<.012\right)$ than the women in the screen negative group.

\subsection{Validation interview outcomes}

A total of 416 previously screened participants (men= 179; women=237) completed the NESHI interview and reported current height, weight, and age so that RMR could be calculated. Of these 179 men, 102 (57.0\%) met criteria for validated night eating. Of the 237 women, 145 (61.2\%) had validated night eating. The breakdown of participants meeting criteria for nocturnal ingestions only, evening hyperphagia only, or both is presented in Table 2 .

Means (SD) for age at interview, current BMI, education, and marital status for men and women by validated night eating group are presented in Table 3. No differences between the positive and negative validated groups emerged in either

Table 1

Demographic information by screened night eating group and sex.

\begin{tabular}{|c|c|c|c|c|}
\hline & \multicolumn{2}{|c|}{ Men } & \multicolumn{2}{|c|}{ Women } \\
\hline & $\begin{array}{c}\text { Negative Screened } \\
\text { Night Eating }\end{array}$ & $\begin{array}{l}\text { Positive Screened } \\
\text { Night Eating }\end{array}$ & $\begin{array}{c}\text { Negative Screened } \\
\text { Night Eating }\end{array}$ & $\begin{array}{c}\text { Positive Screened } \\
\text { Night Eating }\end{array}$ \\
\hline & Mean (SD) & Mean (SD) & Mean (SD) & Mean (SD) \\
\hline Age at Interview (years) & $32.6(8.3)$ & $33.4(8.3)$ & $33.5(7.9)$ & $33.8(8.2)$ \\
\hline Current BMI $\left(\mathrm{kg} / \mathrm{m}^{2}\right)$ & $\begin{array}{l}25.4(4.3) \\
\quad \mathrm{N}(\%)\end{array}$ & $\begin{array}{l}25.7(4.4) \\
\quad \mathrm{N}(\%)\end{array}$ & $\begin{array}{c}23.1(4.0) \\
\mathrm{N}(\%)\end{array}$ & $\begin{array}{l}24.6(5.4) \\
\quad \mathrm{N}(\%)\end{array}$ \\
\hline $\begin{array}{l}\text { Less than College } \\
\text { Education }\end{array}$ & $56(65.1 \%)$ & $58(62.4 \%)$ & $49(49.5)$ & $91(65.9)$ \\
\hline Married/Cohabiting & $49(57.0)$ & $50(53.8)$ & $54(55.1)$ & $83(61.0)$ \\
\hline
\end{tabular}


Table 2

Number (\%) of those with validated night eating endorsing validated nocturnal ingestions and validated evening hyperphagia.

\begin{tabular}{lcc}
\hline & Men $(\mathrm{n}=102)$ & Women $(\mathrm{n}=145)$ \\
\hline Nocturnal Ingestions Only & $8(7.8)$ & $9(6.2)$ \\
Evening Hyperphagia Only & $70(68.6)$ & $104(71.7)$ \\
Nocturnal Ingestions and & $8(7.8)$ & $17(11.7)$ \\
$\quad$ Evening Hyperphagia & $16(15.7)$ & $15(10.3)$ \\
Evening Hyperphagia - & & \\
$\quad$ Missing Nocturnal Ingestions & & \\
\hline
\end{tabular}

men or women for age at interview (men: $\mathrm{t}$-value $=-0.82$, $\mathrm{p}<.42$; women: $\mathrm{t}$-value $=0.31, \mathrm{p}<.77)$, BMI (men; t-value $=$ 1.23, $\mathrm{p}<.23$; women: $\mathrm{t}-\mathrm{value}=1.43, \mathrm{p}<.16$ ), the number married/cohabiting (men; $\chi^{2}=0.18, \mathrm{p}<.67$; women: $\chi^{2}=$ $0.55, \mathrm{p}<.46$ ), or the number with a college education (men: $\chi^{2}=1.55, \mathrm{p}<.22$; women: $\chi^{2}=0.20, \mathrm{p}<.66$ ).

Night eating symptoms for both validated night eating groups by sex, as assessed by the NESHI, are itemized in Tables 4 and 5. For men, symptoms with the greatest differentiation between the negative and positively validated night eating groups were increased morning anorexia, evening/nighttime cravings, insomnia, needing to eat to sleep, distress, and impairment in the positively validated group. For women, the pattern was somewhat different, with more frequent morning anorexia, insomnia, needing to eat to sleep, distress, and lack of control of evening eating being higher in the positively validated group.

\subsection{Full night eating syndrome}

A total of eight participants had insufficient information to determine a threshold NES diagnosis. Three of 175 (1.7\%) men and $11(4.7 \%)$ of 233 women met the threshold NES criteria. Including distress or impairment in the definition reduced these numbers to 0 men and $6(2.6 \%)$ women.

\subsection{Validation of screening questions}

\subsubsection{Men}

Comparing the information from the screening questions with the information from the validation instruments, a total of 147 men had complete information for the screening and interview items: 35 (23.8\%) were correctly classified as nonnight eaters (negative screen, negative interview) and 53
Table 4

$\mathrm{N}(\%)$ of men who endorsed night eating symptoms by validated nighteating group.

\begin{tabular}{lcclll}
\hline & \multicolumn{2}{c}{$\begin{array}{c}\text { Negative Validated } \\
\text { Night Eating }\end{array}$} & & \multicolumn{2}{c}{$\begin{array}{c}\text { Positive Validated } \\
\text { Night Eating }\end{array}$} \\
\cline { 2 - 3 } \cline { 6 - 7 } & No & Yes & & No & Yes \\
\hline Morning Anorexia & $54(70.1)$ & $23(29.9)$ & & $67(65.7)$ & $35(34.3)$ \\
Evening/Night Cravings & $41(53.2)$ & $36(46.8)$ & & $70(68.6)$ & $32(31.4)$ \\
Insomnia & $60(77.9)$ & $17(22.1)$ & & $58(56.9)$ & $44(43.1)$ \\
Eat to Sleep & $25(96.2)$ & $1(3.8)$ & & $43(78.2)$ & $12(21.8)$ \\
Evening Depression & $54(70.1)$ & $23(29.9)$ & & $74(72.6)$ & $28(27.4)$ \\
Distress & $3(100.0)$ & $0(0.0)$ & & $19(82.6)$ & $4(17.4)$ \\
Impairment & $3(100.0)$ & $0(0.0)$ & & $19(82.6)$ & $4(17.4)$ \\
Awareness & $0(0.0)$ & $6(100.0)$ & & $1(3.3)$ & $29(96.7)$ \\
Evening Control & $21(27.3)$ & $56(72.7)$ & & $31(30.4)$ & $71(69.6)$ \\
Night Control & $6(100.0)$ & $0(0.0)$ & & $28(93.3)$ & $2(6.7)$ \\
\hline
\end{tabular}

Not all items were asked to all participants, as there are stop criteria built into the Night Eating Syndrome History and Inventory (NESHI). For example, if a participant did not report awakenings from sleep, they were not asked whether they believed they needed to eat to fall back to sleep during the night or their level of awareness during nocturnal eating episodes. Evening/night cravings assessed how strong participants' cravings were in the evening and when up during the night. If they responded positive to either, they were included in the positive group. Evening and night control assessed how much control participants felt over their eating in the evening and nighttime.

(36.1\%) were correctly classified as night eaters (positive screen, positive interview). Thirty-two (21.8\%) were misclassified as non-night eaters (negative screen, positive interview). Twenty-seven (18.4\%) were misclassified as night-eaters (positive screen, negative interview). These outcomes yielded a positive predictive value $=.66$, a negative predictive value $=.52$, sensitivity $=.62$, and specificity $=.56$ for the screening questions among men.

\subsubsection{Women}

For the 205 women who had complete responses for the screening and interview items, 39 (19.0\%) were correctly classified as non-night eaters (negative screen, negative interview) and 80 (39.0\%) were correctly classified as night eaters (positive screen, positive interview). There were $47(22.9 \%)$ who were misclassified as non-night eaters (negative screen, positive interview) and $39(19.0 \%)$ misclassified as night-eaters (positive screen, negative interview). These outcomes yielded a positive

Table 3

Body size and socio-demographic information by validated night eating group and sex.

\begin{tabular}{|c|c|c|c|c|}
\hline & \multicolumn{2}{|c|}{ Men } & \multicolumn{2}{|c|}{ Women } \\
\hline & $\begin{array}{l}\text { Negative Validated } \\
\text { Night Eating }\end{array}$ & $\begin{array}{l}\text { Positive Validated } \\
\text { Night Eating }\end{array}$ & $\begin{array}{l}\text { Negative Validated } \\
\text { Night Eating }\end{array}$ & $\begin{array}{l}\text { Positive Validated } \\
\text { Night Eating }\end{array}$ \\
\hline & Mean (SD) & Mean (SD) & Mean (SD) & Mean (SD) \\
\hline Age at Interview (years) & $32.5(8.5)$ & $33.5(8.1)$ & $33.9(7.8)$ & $33.6(8.2)$ \\
\hline \multirow[t]{2}{*}{ Current BMI $\left(\mathrm{kg} / \mathrm{m}^{2}\right)$} & $26.0(4.6)$ & $25.2(4.1)$ & $24.5(4.5)$ & $23.6(5.2)$ \\
\hline & $\mathrm{N}(\%)$ & N (\%) & $\mathrm{N}(\%)$ & $\mathrm{N}(\%)$ \\
\hline Less than College & $53(68.8)$ & $61(59.8)$ & $56(60.9)$ & $84(57.9)$ \\
\hline Married/Cohabiting & $44(57.1)$ & $55(53.9)$ & $56(61.5)$ & $81(56.6)$ \\
\hline
\end{tabular}


Table 5

$\mathrm{N}(\%)$ of women who endorsed night eating symptoms by validated nighteating group.

\begin{tabular}{lccccc}
\hline & \multicolumn{2}{c}{$\begin{array}{c}\text { Negative Validated } \\
\text { Night Eating }\end{array}$} & & \multicolumn{2}{c}{$\begin{array}{c}\text { Positive Validated } \\
\text { Night Eating }\end{array}$} \\
\cline { 2 - 3 } \cline { 6 - 7 } & No & Yes & & No & Yes \\
\hline Morning Anorexia & $66(71.7)$ & $26(28.3)$ & & $94(64.8)$ & $51(35.2)$ \\
Evening/Night Cravings & $60(65.2)$ & $32(34.8)$ & & $104(71.7)$ & $41(28.3)$ \\
Insomnia & $45(48.9)$ & $47(51.1)$ & & $55(37.9)$ & $90(62.1)$ \\
Eat to Sleep & $53(100.0)$ & $0(0.0)$ & & $94(86.2)$ & $15(13.8)$ \\
Evening Depression & $42(45.6)$ & $50(54.4)$ & & $69(47.6)$ & $76(52.4)$ \\
Distress & $5(71.4)$ & $2(28.6)$ & & $23(62.2)$ & $14(37.8)$ \\
Impairment & $5(71.4)$ & $2(28.6)$ & & $29(78.4)$ & $8(21.6)$ \\
Awareness & $0(0.0)$ & $8(100.0)$ & & $2(4.8)$ & $40(95.2)$ \\
Evening Control & $24(26.1)$ & $68(73.9)$ & & $60(41.4)$ & $85(58.6)$ \\
Night Control & $7(87.5)$ & $1(12.5)$ & & $38(90.5)$ & $4(9.5)$ \\
\hline
\end{tabular}

Not all items were asked to all participants, as there are stop criteria built into the Night Eating Syndrome History and Inventory (NESHI). For example, if a participant did not report awakenings from sleep, they were not asked whether they believed they needed to eat to fall back to sleep during the night or their level of awareness during nocturnal eating episodes.

predictive value $=.67$, a negative predictive value $=.45$, sensitivity $=.63$, and specificity $=.50$ for the screening items among women.

\subsection{Coherence of NES symptoms}

Conditional probabilities were calculated to examine the co-occurrence of NES behavioral symptoms based on interview data. Values are presented in Tables 6 and 7, showing the probability of reporting symptoms presented in the far left column, given that symptoms presented in the top row were confirmed. Any value over .50 is a greater than $50 \%$ chance. For example, if a woman met criteria for evening hyperphagia, she had a $10 \%$ chance of also meeting criteria for nocturnal ingestions, but a $60 \%$ chance of meeting criteria for insomnia; further, if a woman met criteria for nocturnal ingestions, she had a $65 \%$ chance of also meeting criteria for evening hyperphagia, and a $96 \%$ chance of meeting criteria for insomnia.

The relative strength of the co-occurrence of symptoms differed for men (Table 6) and women (Table 7). Among men, meeting criteria for evening hyperphagia did not produce any probabilities of co-occurrence with other symptoms greater than .38. The presence of nocturnal ingestions more often co-occurred with evening hyperphagia and the belief in the need to eat to fall back to sleep, both at .50 . The presence of this belief co-occurred most often with other night eating symptoms for men. Of the 42 possible cooccurring symptoms, 8 were calculated to be $\geq .50$ for men.

For women the conditional probabilities were generally higher than for men, particularly in the presence of nocturnal ingestions and the belief in the need to eat to fall asleep. Still, the presence of evening hyperphagia did not seem as highly related as nocturnal ingestions to many of the night eating symptoms, with the exception of insomnia (.60) and depressed mood in the evening (.52). Of the 42 possible co-occurring symptoms, 21 were calculated to be $\geq .50$ for women.

\section{Discussion}

The use of screening questions in epidemiological studies is common, yet little is known about how results from screening compare with diagnosis outcomes from clinical interviews. In the current study, two screening questions for identifying night eating behaviors produced modest estimates of sensitivity (.62 for men and .63 for women) and specificity (.56 for men and .50 for women). These parameters suggest that almost two-thirds of participants were correctly identified as having NES (without considering distress or impairment) if they answered positively on the screening questions, and about half of the participants were correctly identified as not having NES when answering negatively on the screening questions. These results are strikingly similar for both men and women and indicate that clinical interviews remain the gold standard for identifying clinical cases, in this instance, for NES.

According to the original screening data, more men than women screened positive for both broad and narrow definitions of night eating $(4.6 \%$ and $2.5 \%$ for men vs. $3.4 \%$ and $1.7 \%$ for women, respectively) [11]. After interview, slightly more cases of validated night eating (based on Criterion A) were found for women (61\%) than men $(57 \%)$. Also, BMI was positively associated with screened cases of night eating, but this relationship was not

Table 6

Conditional probabilities in men for reporting symptoms in the far left column, given that symptoms presented in the top row were reported.

\begin{tabular}{|c|c|c|c|c|c|c|c|}
\hline & $\begin{array}{l}\text { Evening } \\
\text { Hyperphagia }\end{array}$ & $\begin{array}{l}\text { Nocturnal } \\
\text { Ingestions }\end{array}$ & $\begin{array}{l}\text { Morning } \\
\text { Anorexia }\end{array}$ & $\begin{array}{l}\text { Evening/Night } \\
\text { Cravings }\end{array}$ & Insomnia & $\begin{array}{l}\text { Eat to } \\
\text { Sleep }\end{array}$ & $\begin{array}{l}\text { Low Evening } \\
\text { Mood }\end{array}$ \\
\hline Evening Hyperphagia & - & .50 & .55 & .41 & .59 & .54 & .49 \\
\hline Nocturnal Ingestions & .10 & - & .14 & .12 & .27 & .62 & .14 \\
\hline Morning Anorexia & .34 & .44 & - & .40 & .30 & .31 & .20 \\
\hline $\begin{array}{c}\text { Evening/Night } \\
\text { Cravings }\end{array}$ & .30 & .44 & .47 & - & .41 & .54 & .35 \\
\hline Insomnia & .38 & 1.00 & .31 & .37 & - & .85 & .41 \\
\hline Eat to Sleep & .15 & .50 & .14 & .21 & .21 & - & .24 \\
\hline Low Evening Mood & .27 & .37 & .17 & .26 & .34 & .46 & - \\
\hline
\end{tabular}


Table 7

Conditional probabilities in women for reporting symptoms in the far left column, given that symptoms presented in the top row were reported.

\begin{tabular}{|c|c|c|c|c|c|c|c|}
\hline & $\begin{array}{l}\text { Evening } \\
\text { Hyperphagia }\end{array}$ & $\begin{array}{l}\text { Nocturnal } \\
\text { Ingestions }\end{array}$ & $\begin{array}{l}\text { Morning } \\
\text { Anorexia }\end{array}$ & $\begin{array}{l}\text { Evening/Night } \\
\text { Cravings }\end{array}$ & Insomnia & Eat to Sleep & $\begin{array}{l}\text { Low Evening } \\
\text { Mood }\end{array}$ \\
\hline Evening Hyperphagia & - & .65 & .62 & .52 & .60 & .73 & .56 \\
\hline Nocturnal Ingestions & .14 & - & .15 & .20 & .19 & .87 & .14 \\
\hline Morning Anorexia & .35 & .38 & - & .41 & .34 & .20 & .37 \\
\hline Evening/Night Cravings & .28 & .50 & .39 & - & .31 & .73 & .27 \\
\hline Insomnia & .60 & .96 & .60 & .57 & - & .93 & .65 \\
\hline Eat to Sleep & .11 & .50 & .06 & .22 & .11 & - & .10 \\
\hline Low Evening Mood & .52 & .62 & .61 & .47 & .60 & .60 & - \\
\hline
\end{tabular}

observed for validated cases of night eating. Participants meeting Criteria $\mathrm{A}, \mathrm{B}$, and $\mathrm{C}$ reduced these estimates substantially to $1.7 \%$ of men and $4.7 \%$ of women. When including the distress and impairment in functioning as required elements for diagnosis, no men and 3\% of women remained. The reduction in those meeting each successively restrictive criterion highlights important sex differences. Men reported the full constellation of night eating symptoms less often than women and did not report distress or impairment.

These data also allow us to examine the utility and coherence of individual items of the proposed diagnostic criteria [4]. Items more often reported among those with validated night eating for both men and women were morning anorexia, insomnia, and the belief in the need to eat to sleep, with the latter two showing the most differentiation. The rates of endorsement for the remaining items were intriguingly similar between those with and without validated night eating. Previous work using item response theory analysis showed that, aside from the core criteria, initial insomnia and nighttime awakenings were most informative in discriminating persons with night eating [21]. That study did not include the belief in needing to eat to sleep in its analysis. It seems that items most closely related to nocturnal ingestions are most predictive of the night eating construct.

More evidence of this is provided with the conditional probabilities analysis. Cohesion of symptoms has been argued to be a necessary element of a psychiatric disorder [see 22,23 ], typically suggesting that there should be a $50 \%$ chance if one diagnostic criterion is met, that another will be fulfilled [24]. The presence of nocturnal ingestions met this standard with several other night eating symptoms, including the presence of evening hyperphagia. However, evening hyperphagia did not meet the $50 \%$ criteria with other symptoms for men, and only with insomnia and depressed mood in the evening for women. In fact, among men, very few symptoms co-occurred at a rate of $50 \%$ or more, suggesting the night eating behaviors, particularly evening hyperphagia, often occurred in isolation in this group. Among women, symptoms more often occurred at a $\geq 50 \%$ chance, suggesting that the symptoms were more interrelated in this group. Lundgren and colleagues [20] showed different results among a sample of inpatients with other eating disorders. In that group, evening hyperphagia was more likely to be related to the remaining night eating symptoms, but not as strongly as the presence of nocturnal ingestions. It seems likely that with increasing psychiatric pathology, more symptoms co-occur.

Results of this first validation study of night eating screening questions against interview-based diagnoses must be considered within the context of its limitations. First, substantial attrition of participants at each successive step in the analysis is likely related to the burden of the STAGE study. This attrition may introduce bias in the results. Second, the time lag between the collection of screening data and the execution of the interviews, due mainly to the logistics of coordinating this international effort, may have allowed time for symptoms to change, thus influencing our ability to confirm the validity of the screening questions. Third, food recall for the evening hyperphagia criterion was limited to all foods consumed between $8 \mathrm{pm}$ and $5 \mathrm{am}$. This was done primarily to reduce burden on the participants but also because the interviewers were not dietitians or mental health professionals, and the starting time for these recalls varied widely. However, this is the time period used in previous clinical studies if a clear "dinner" meal could not be discerned from food diaries [e.g., 25,26].

The RMR calculation was based on this recall to produce an estimate of evening hyperphagia, which might lead to a greater number of participants meeting the caloric intake requirement for night eating. To test this possibility, we performed the same transformation of energy intake data based on RMR from a pre-existing dataset where 24-h food intake data were available from one-week food journals [27]. The percent of actual total calories consumed between 8:00 pm and 5:00 am from the Boston et al. data was calculated, and the participants $(n=148)$ were grouped into those who consumed $25 \%$ or more of their calories at night and those who did not. Tetrachoric correlations were calculated comparing the evening hyperphagia grouping based on RMR and the grouping based on percent of total caloric intake. For men, this value was .92, for women, it was .86 , lending support to our use of the RMR to define evening hyperphagia in this sample.

Although participants in this study were related, the responses to the screening questions and the validity of the screening tools should not be influenced by the non-independence of the sample. The effects of non- 
independence are generally observed when evaluating associations among variables by increasing the probability of making a Type 1 error if adjustments are not made for the non-independence. The nature of this study did not require such adjustments. Finally, we do not know if participants who reported nocturnal ingestions were waking specifically to eat, or were awake because of primary insomnia and ate for other reasons. This motivational issue is an important one to pursue in future studies.

In sum, the validity of screening questions in a large population-based study to identify cases of night eating was modest. While using screening items is unavoidable in many large-scale studies, interviews remain the gold standard. Additionally, night eating symptoms with the most utility for differentiating the diagnosis in this sample were insomnia and the belief in the need to eat to fall asleep. As the discussion regarding a stable and coherent set of diagnostic criteria continues, it is important to note that the evening hyperphagia threshold was met commonly in this sample, but occurred frequently in isolation from other criteria, while the presence of nocturnal ingestions was more coherent with other symptoms. Women also presented with more coherent symptoms of night eating than men, who did not indicate distress for their symptoms. Future studies should compare validity of screening questions and symptom co-occurrence in various populations, including treatment seeking samples, as symptom coherence in those individuals may differ from community samples. Based on our findings, future epidemiologic studies would benefit from assessing both nocturnal ingestions and evening hyperphagia, along with initial insomnia and the belief that one needs to eat to sleep when screening for NES. Further research should confirm this finding along with further examination of the clinical utility of the proposed diagnostic criteria for NES.

\section{Acknowledgment}

This study was supported by grants awarded to Kelly C. Allison (University Research Foundation, University of Pennsylvania) and Finn Rasmussen (Swedish Council for Working Life and Social Research, grant number 20050399). We thank the Swedish Twin Registry for their support of this study.

Portions of this study were presented at the International Conference on Eating Disorders, 2011 in Miami, Florida.

\section{References}

[1] Stunkard AJ, Grace WJ, Wolff HG. The night-eating syndrome: a pattern of food intake among certain obese patients. Am J Med 1955; 19:78-86.

[2] American Psychiatric Association. Diagnostic and statistical manual of mental disorders: DSM 5. Washington, DC: American Psychiatric Association; 2013.

[3] World Health Organization International Classification of Diseases (ICD). The ICD-10 classification of mental and behavioral disorders: clinical descriptions and diagnostic guidelines. http://www.who.int/ classifications/icd/en.

[4] Allison KC, Lundgren JD, O’Reardon JP, Geliebter A, Gluck M, Vanai $\mathrm{P}$, et al. Proposed diagnostic criteria for night eating syndrome. Int $\mathrm{J}$ Eat Disord 2010;43:241-7.

[5] American Academy of Sleep Medicine. International classification of sleep disorders: diagnostic and coding manual. 2nd ed. Westchester, IL: American Academy of Sleep Medicine; 2005.

[6] Allison KC, Lundgren JD, O'Reardon JP, Martino NS, Sarwer DB, Wadden TA, et al. The Night Eating Questionnaire (NEQ): psychometric properties of a measure of severity of the night eating syndrome. Eat Behav 2008;9:62-72.

[7] Moizé V, Gluck ME, Torres F, Andreu A, Vidal J, Allison KC. Transcultural adaptation of the Night Eating Questionnaire (NEQ) for its use in the Spanish population. Eating Behav 2012;13:260-3.

[8] Harb AB, Caumo W, Hidalgo MP. Translationand adaptation of the Brazilian version of the Night Eating Questionnaire. Cad Saude Publica 2008;24:1368-76.

[9] Rand CSW, Macgregor AMC, Stunkard AJ. The night eating syndrome in the general population and among postoperative obesity surgery patients. Int J Eat Disord 1997;22:65-9.

[10] Striegel-Moore RH, Dohm FA, Hook JM, Schreiber GB, Crawford PB, Daniels SR. Night eating syndrome in young adult women: prevalence and correlates. Int J Eat Disord 2005;37:200-6.

[11] Lamerz A, Kuepper-Nybelen J, Bruning N, Wehle C, Trost-Brinkhues $\mathrm{G}$, Brenner $\mathrm{H}$, et al. Prevalence of obesity, binge eating and night eating in a cross sectional field survey of 6-year-old children and their parents in a German urban population. J Child Psychol Psychiatry 2005;46:385-93.

[12] Striegel-Moore RH, Franko DL, Thompson D, Affenito S, May A, Kraemer HC. Night eating: prevalence and demographic correlates. Obesity 2006;14:139-47.

[13] Tholin S, Lindroos A, Tynelius P, Akerstedt T, Stunkard AJ, Bulik $\mathrm{CM}$, et al. Prevalence of night eating in obese and nonobese twins. Obesity 2009;17:1050-5.

[14] Striegel-Moore RH, Franko DL, Garcia J. The validity and clinical utility of night eating syndrome. Int J Eat Disord 2009;42:720-38.

[15] Lundgren JD, Allison KC, Vinai P, Gluck ME. Assessment instruments for night eating syndrome. In: Lundgren JD, Allison KC, \& Stunkard AJ, editors. Night eating syndrome: definition, assessment, and treatment. New York: Guilford Press; 2012. p. 197-217.

[16] Lichtenstein P, Sullivan PF, Cnattingius S, Gatz M, Johansson S, Carlström E, et al. The Swedish Twin Registry in the third millennium: an update. Twin Res Hum Genetics 2006;9:875-82.

[17] Furberg H, Lichtenstein P, Pedersen NL, Thornton L, Bulik CM, Lerman C, et al. The STAGE cohort: a prospective study of tobacco use among Swedish twins. Nicotine Tobacco Res 2008;10:1727-35.

[18] Mifflin ST, Hill LA, Scott BJ, Daugherty SA, Koh YO. A new predictive equation for resting energy expenditure in healthy individuals. Am J Clin Nutr 1990;51:241-7.

[19] Frankenfield D, Roth-Yousey L, Compher C, for the Evidence Analysis Working Group. Comparison of predictive equations for resting metabolic rate in healthy nonobese and obese adults: a systematic review. J Am Diet Assoc 2005;105:775-89.

[20] Lundgren JD, McCune A, Spresser C, Harkins P, Zolton L, Mandal K. Night eating patterns of individuals with eating disorders: implications for conceptualizing the night eating syndrome. Psychiatry Res 2011;186:103-8.

[21] Allison KC, Engel SG, Crosby RD, de Zwaan M, O'Reardon JP, Wonderlich SA, et al. Evaluation of diagnostic criteria for night eating syndrome using item response theory analysis. Eat Behav 2008;9:398-407.

[22] Striegel-Moore RH, Franko DL, May A, Ach E, Thompson D, Hook JM. Should night eating syndrome be introduced in the DSM? Int J Eat Disord 2006;9:544-9.

[23] Stunkard AJ, Allison KC, Geliebter A, Lundgren JD, Gluck ME, O'Reardon JP. Development of criteria for a diagnosis: lessons 
from the night eating syndrome. Compr Psychiatry 2009;50: 391-9.

[24] Blashfield RK, Sprock J, Fuller AK. Suggested guidelines for including or excluding categories in the DSM-IV. Compr Psychiatry 1990;31:15-9.

[25] Allison KC, Lundgren JD, Moore RH, O'Reardon JP, Stunkard AJ. Cognitive behavior therapy for night eating syndrome: a pilot study. Am J Psychotherapy 2010;64:91-106.
[26] O'Reardon JP, Ringel BL, Dinges DF, Allison KC, Rogers NS, Martino NS, Stunkard AJ. Circadian eating and sleeping patterns in the night eating syndrome. Obes Res 2004;12: 1789-96.

[27] Boston RC, Moate PJ, Allison KC, Lundgren JD, Stunkard AJ. Modeling circadian rhythms of food intake by means of parametric deconvolution: results from studies on the night eating syndrome. Am J Clin Nutr 2008;8:1672-7. 